\title{
A Survey on Location based Services and Positioning Techniques
}

\author{
Moein Mohammadi \\ Department of Computer \\ Science, Shahid Bahonar \\ University, Kerman, Iran
}

\author{
Ehsan Molaei \\ Department of Computer \\ Science, Shahid Bahonar \\ University, Kerman, Iran
}

\author{
Ali Naserasadi \\ Industrial and Mining Faculty, \\ Shahid Bahonar University, \\ Kerman, Iran
}

\begin{abstract}
Ever increasing grows of mobile links and need to new technologies in many industries and other job like tourism. Taxi services, could compensate this blank with location based service (LBS) and introduce as public example of GIS in the world. Therefore, in the present article, we consider the concepts, application, component and other information of it by most valid resource.
\end{abstract}

\section{General Terms}

Location Based Services

\section{Keywords}

Location Based Services, Mobile commerce, Data Transfer Network, Positioning, Global Positioning System

\section{INTRODUCTION}

Human always to follow live in more prosperity and well- being from the beginning up to now, and try to a chive them. Therefore, we could introduce some new technology such as internet and mobile links which have great effects on communication and life's of people, so that with growing of mobile links and increasing of subscribers and also more policing of GPS services to be found new idea in the field of publicities, marketing, civil services and etc. which one of them was starting a thing as a location based services.

The main idea of this think is explicit. Pocket- size computers like mobile, tablet, PDK and etc. which are increasingly from the view point of power of increasing and computation become a pretext that think to them as application of main purposes. This means that it is not necessary, for example for mobile that use only for communication and also could use of it as GPS or publicity and marketing and or other services.

You suppose, for example, a person who is walking in a city and decide go to a restaurant for dinner and eat specific food. Now, one fast (not best) method of searching all restaurants and their menu is in the internet and by mobile. Does this method is intellectual? A best method is providing restaurant which located near the person and this work is possible only with new techniques at LBS.

An official definition of LBS is available information services of mobile by mobile links and using the ability of determining the positioning mobile. [1]

LBS is a common point between some techniques by this definite which are including geographical information system(GIS) and internet and long distance communication system and NISTs which are show in figure 1.[1]
The first generation of LBS steps onto the arena at 1997, and continue till 2001 which enacted more specific standards for LBS and create a new generation LBS systems after that.

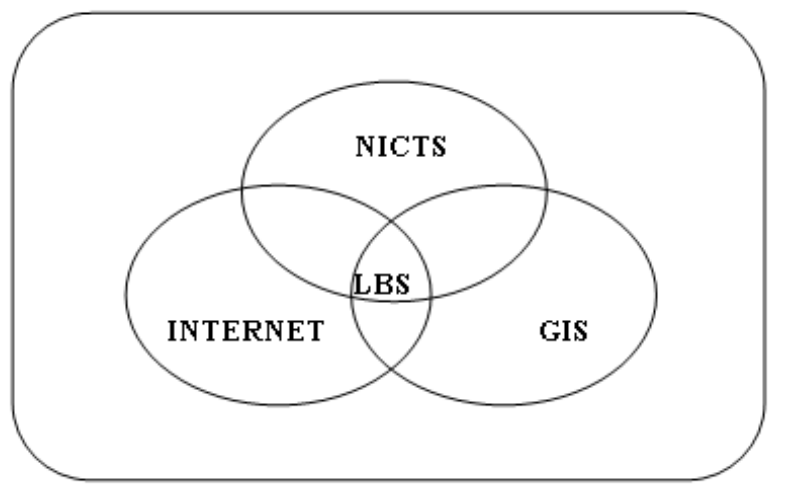

Figure 1: LBS as an intersection of technologies [2]

\section{MOBILE COMMERCE}

Internet technology development, link and growing the personal mobile set leading to increase growing mobile business and mcommerce. Mobile set are small and light as well as power full and increasingly are used for manual computational, and always carry by their owners. In addition, tracking the position of terminal by using satellite orientation system at mobile links causes the development and unity of wireless devices position and provides using of position based services. New smart telephones are programmable and are capable to performance application program. Services based on position are specific mode of mobile business and often use real position of terminals in providing services.

The range of position based services are including player as a telephone user, transmission operator, services provider and etc. [3]

\section{LBS HISTORY AND GENERATIONS}

The first generation of LBS starting by service central points at ss7 link between $1997-2001$, which these points determine the position of mobile by using link. These points are famous at Is41 like to MPC (Mobile positioning center) and GSM, UTMS to GMLC (Gateway Mobile location center).

The first generation was according to provider operator of link services which create connection by operation to available to MPC/GMLC and use applicable program. The first problem of this issue was lack of any standard connection to communicating applicable program with MPC/ GMLC. The second problem 
was lack distribution systems and usually explain instantaneous. Also, this is included time aspect which indicates to system changes by passing of time and need to compatibility of pre and post mode of system's change. The third problem was lack of comprehensive and unity in this system when operator's link will to provide new service by using written applicable program to own users, he / she should be do all care full performance with high price, because unity of system will be kept after provide new services. Existing problems is first phase leading to move LBS to crating second phase. New phase are included two new cases in comparable with first phase: location - enabling middle ware and Geo server. The first case is used as a main core system in new architecture and satisfies many important needs. This concentrated structure is caused of uniting location of system.

In the second case, applicable program developer used standard APZ functions and this means that they use the ability and options of two cases in their own program. Second generation of standards plays important role in architecture. Two important standards which are based on XML are included MLP (Mobile location protocol) and openls (open location service). The first standards are used as a connection between servers like MPC and GMCC and location - enabling middleware server. This connection is included a serves question and answer between two server and it takes points position of MPC/ GMLC server and return it to requested server and finally application program [2].

\section{GENERAL BASIC AT LBS}

We consider general discussion and definite of component in relation to location based services in this section and understand the application of these systems.

\subsection{Concept of LBS}

GIS and LBS have similarities. One of them is using source date and analyzed functions and data analyzed. Therefore, GIS and LBS commonly are able to answer the question like that:

1. Where is the position of user?

2. What are in the proximity of the user?

3. How user could be achieving to their purpose?

But, there are different in these two systems. GIS is oldest and known as a professional system which in used by experienced user with diverse set of analyzed tools of location data. The, GIS need power process ability and computational resources. In comparison, LBS could only provide limited services to nonprofessional user. Therefore, these systems usually face with problem such as low process ability at telephone and pay affection to limited energy resources in this devise [14].

\subsection{Classification of LBS based on the Operations}

Positioning: the services of positioning to provide the available in formation and consider the resource on the basic of where is user bow? A simple example of location based, for example a map of streets or weather which is centralized itself with the current position of user.

Tracking: Tracking service is generalization of positioning services which related to providing information resources on the basic of current and before position of user.
Mobile resource Allocation: this service is generalization of tracking service which is related to providing information and resources on the basic of current and before position of user and also designing whether the user when is need to be in the future. An example of mobile resource allocation is navigation like guiding the position of tourist [3].

\subsection{The Components of LBS}

Mobile tools: It is tools user requested information by system. The result of this request could be audio, pictorial, and textual. PDA (pocket digital assistant), mobile sets and laptop are examples of these tools. While, navigation part of a device could be one of this tools.

Communication link: second part is mobile link which is communicated between user and provider of the system. Such a way that data and user request is delivering to user cont then the results will return to user.

Device of positioning: This system often to provide services, and positioning information of user. Positioning of user could be done by methods of positioning at wireless link or by using GPS if there exist some services of positioning. In the case of lacking one of these systems, user can enter his / her position by manual.

Service provider: LBS providers usually provide different services to user in the result of processing her/his request. This services are included positioning, user's address, lining a way to distinction point, returning interested information of user and etc.

Data and content $\mathbf{t}$ provider: services provider usually does not save the information of user by own, but take the collecting information of geographical data and positioning information from the companies and then them to the user after process. The sample of this provider is traffic information or map making company. [2]

These components are showed in figure 2 .

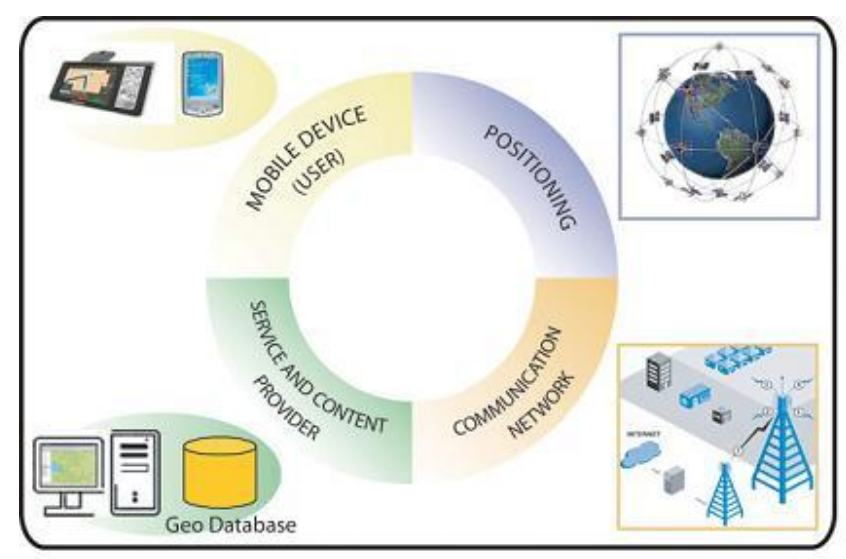

Figure 2.The basic components of LBS [2]

\subsection{Main Acts on Data at LBS}

There are five main and important acts on positioning data by attention to needs of user:

1. Locating: the most important question is that know that where is user by to a person or specific place?

2. Searching: A user may be finding to search person, object or specific event. 
3. Navigating: In means that search a way to distinction point.

4. Identifying: searching the properties and characteristics of specific place.

5. Checking: events which occur in a specific place.

Now, we consider the needs of these five main acts.

Locating and navigation acts are based on a positioning data. But other three act need to different information. In addition to positioning information, we need this information.

1. Comprehensive and complete information which are constant. Examples of this information are in yellow page book. These types of information usually are not change by passing of time and also are available by other media such as newspaper, map, internet and etc.

2. Topical and located information which are change when user move. In such case, provided information are not valid. The examples of this information are traffic information, weather fore cast, information about the tickets of theater or open and closing of a restaurant.

3. Immunity information which have specific importance. For example, information about roads condition, weather change and landslide risk which are included immunity problem. Also, in emergency condition, user like drivers of car needs information like first aids or small repairing of car.

4. Personal information of user: In most times. Ask opinion only as a consumer of information, while, opinion polling and her/ his interested have better result. These information are like interest places of user to visit, her/ his interest to specific event for example concert and using user's criterion at searching best way (shortest time and distant).

It is necessary to note that checking act is use as events property as a positioning and time information [2].

\section{TYPE OF SERVICES AT LBS}

In general, we could distinguish between two groups of LBS service in according to information which are used in communicate with user or not.

1. Pull services: pull services are deliver information to user request directly. This act is similar to see a web page in internet and entering its address on the internet's search. These types of services are two groups: Functional services such as calling a taxi or ambulance only with pushing a button or information services such as searching near hotel.

2. Push services: These types of service provide information for user that he/ she does not requested or is requested indirectly. These services are usually active by an event which this event is a specific time or access of user to specific limited. For example for services which requested is news services which give information to user when events occurs. An example for service which does not request is advertising messages. This type of service are complex in compare with pervious and system should be available positioning user information as well as his / her interested and preference. The personal rights are very important in this type of services [4].

\section{POSITIONIG AT LBS}

The main component of provider system of positioning is location based services so we will consider it by detailer.

\subsection{Positioning Technology}

Positioning technology is derided in two groups in general: Handset - centric and network - centric, and we will explain each of them.

\subsubsection{Positioning Technology (Handset-Centric) 1. CELL-ID}

CELL-ID is work at WCDMA and GPRS and G\&M lines. This technology need link, so that, the BTS (main station) which is connected to cell phone could be recognize position.

Cell -ID position services. Recognize main station position of UE or MS and deliver this information to system's provider. At first, we use cell- ID when which high level of accuracy is not necessary.

If we sue handset to contact, therefore, information about cell site should be updated instantaneously.

To updating information link at a position of handset, link calling tools and force it cover power signal of BTS and this link aware form cell-ID. The accuracy of this method depended to cell size and in many cases could be poor because, diameter of GSM cell is between $2 \mathrm{~km}-20 \mathrm{~km}$. we could achieve to $250 \mathrm{~m}$ accuracy with Pico cell. We could use TA technology and or signal's power to high level of accuracy.

\section{Timing advance (TA)+CELL-ID}

When, terminal receive signal is very important for operation of GSM/GPRS of link. Every movable station is located at a cell with changeable distance from main station, but signal should be receive to the main station exactly when time lots were available.

Therefore, It is necessary to be compatible movable station with main station in a suitable time. Because of each period time of TA for every movable station is depended to time. Because of each period time of TA for every movable station is depended to the distance of it from main station, so, we can use this information to determine the range of remoteness of contact.

The information of TA for cells with radius over $550 \mathrm{~m}$ is unused for high accuracy of positioning. The case of this issue is assessed pro rata for TK related to moving movable station on the basic of Mow many $550 \mathrm{~m}$ is for of movable station to main station.

\section{Strength CELL-ID}

Movable station always measuring signal's power from every main station and provide this information to the main station. This work is such a way that movable station could transfer and receive information from main station which has optimal and suitable signal's power. So that the quality of contact could be improve for final user.

We could computation the position of who is contact by attention to the related information of signal's power and consideration of prices which is reduce by increasing the 
distance between sender and receiver by power of RX signal.

Although, there are actors which limited effectiveness of this method and distances is not only effective factor. The characteristic of ground between sender and receiver have important effect on measuring. If the maternal which from a building was condensed and the floor of contactor were high, so have more negative effect on signal's power. Measuring signal's power or RX is called sometimes as "the result of link's measuring" (NMR) [2, 5, and 6].

\subsubsection{Positioning Technology (Network-Centric)}

There are some technology of measuring with different basic links could be apply to defer mine the positioning of who is contact. In the following, we explain some of main type of it:

\section{E-OTD}

E-OTD applies only for GPRS and GSM links. In GSM link, MS cover transferring signal from some next BTS and measuring time period between GSM from BTS. This difference in time main basic of E-OTD method and apply to determining movable devices.

The accuracy of E- OTD method are function of difference in times of measuring resolutions, shop of next main station and surrounding of this sigil. Moveable handset should be measuring difference in time at least from 3 main stations and support two- dimensional positioning. E- OTD need to the careful time's information.

Measuring units of positioning (LMUS) are need careful time's information in GPRS and GSM links. The most need of this technology is that BTS is observed in link by at least one LMU. In additional, we need specific software to support E-OTD in MS. LMU in GSM/ GPRS links. In this technology, designing of link, following of local regulation where we deal with new sites, cost's of designing, installing, testing and finally maintenance of LMUS link are very important this level of sensitive and complexity ability of operator to support positioning based services do more complex E-OTD being longer the period of time for positioning service's link.

E-OTD has improved performance in compared with CellID, but it need to use LMUS. This work causes we have high price and complex of performance. Also, it needs inter change many of messages to provide positioning information and updating this information continue. These messages are very at A-GPS or Cell- ID and so E-OTD has applied more band width related to this technology. Accuracy influenced by signal's reflection and error of multipart. This system has not accuracy in rural are because it needs at least 3 man station and we have few BTS in furl area.

\section{OT DOA}

OT DOA applies only for WCDMA. OT DA is a version of WCDMA from E-OTD in general. Techniques of OT DOA'S positioning estimate the positioning of one handset by pay attention to the times of receiving signal at UE at least from 3 stations (BTS). The position of handset is define at intersection of at least two hyperbolas, and is by OT DOA of WCDMA after some B. the weakness point is the result of weak at points without at least 3, B, low accuracy at the length of line link. Error of multipart and harmony appears with only one link While WCDMA link is based on CDMA, so to low power and effectiveness use of this. we use communication's band the ability of handset to use stations is limited seriously, and this issue is influenced on accuracy and general performance of OT DOA is worse than E-OTD in many cases.

This method needs high accuracy to support OT DOA position to LMU which is very costly. (AOA):

\section{AOA}

AOA or entering angle apply multi array antenna and try to estimate the direction of entering signal. Therefore, measuring only with one, is limited the resource position of length a line at AOA, IF we have at least two AOA estimate from two antenna at two different position, so, the original position of signal could be determine at intersection of caring line of 2 antenna, usually, we use few AOA'S estimate to improve the accuracy of estimation by additional information the estimation of AOA is often apply with short and decrease ambiguities or even remove them. In some cases which we apply tall baseline, the purpose of it, is improving resolution.

We use algorithms to estimate AOA and use difference in phase or other charlatanistic of signal between antennas next to antenna's array $[7,8]$.

\subsection{Classification of Positioning Techniques}

There are two techniques of utilizing positioning technology which are explained in the following.

\subsubsection{Active}

Positioning techniques of active determine the position on the basic of sender's signals or beacons; and these are divided in two group:

\section{Neighborhood}

Neighborhoods with Beacon signals by all Mobile are used to determine the position of mobile. A mobile links use array of wireless Bender at specific positions to reinforcement and deliberating contact. In preliminary level, every sender will cover small limited geographical which its signals are power than other and it calls as cell. The power of signal will reduce with increase distance from sender. Therefore, in a simple case, shape of cell made and every cell included near position to sender. A mobile with determining sender could specify in which cell by most power signal, and is depended to sending an average of contacts $[17,18]$.

\section{Triangular}

There are two triangular, late ration and angulations; late ration is computational process of object's positioning on the basic of distance from other position. An angulation is computational process of object's positioning on the basic of its angular from other position. GPS system is on the basic of late ration. Angulations method also use in some mobile link to determining position. Some of positioning techniques of Active such as GPS use movable sensor to receiving signals of beacons $[17,18]$. 


\subsubsection{Passive}

Positioning techniques of passive are not basic of sending signals of beacons. These techniques are determined position on the basic of main sensor with related to positioning information about environment indirectly. Geographical environment is very hetero generous.

Therefore often all kind of measuring could be in some positioning passive from and deviled in two groups:

\section{Motion tracking}

To tracking speed of robot at time, we use speed and direction in robots. This information could be applied to computation of a robot position at a specific point of time related to start point. Tracking techniques are called Dead reckoning sometimes.

\section{Scene Analysis}

A digital camera is an array of this, which could be apply to determine object's or person's position in a digital picture or apply to determine camera's position of take a picture $[10,16]$.

\section{LOCATION BASED SYSTEM AND PERSONAL PROBLEMS}

Location based system could be change many of routine. The important challenge of this question is how to be personal problem's of people when they use location based services? All people are interested to aware of automatic technology of emergency services about situations person at emergency. While, if personal information of any person transfer, he/she has not good feel. The limited personal of people is known as a main right and should be memorized, so, this work is called fair information practice or data protection.

The purposes of data protection of organs are:

1. Personal data are collect and use only for specific purposes.

2. Personal data are collected only by own satisfaction.

3. Precaution measures cue do to assure that this personal data are accuracy.

Date protection should be achieved to a balance between protection personal limits of persons and the ability of new technology to development. Data protection is related to a person. But LBS have specific problems for example.

1. Aware of person position could be applied to understand other personal information. For example, what person does or know his/her interested. For example, positional information about repeated consult to the hospital is cause that organ understand that he/she is illness and this understanding have negative effect on work aspect. Many of people know this unfair work, violation of their rights.

2. Location based systems are not good index to determine person's position location based is different in accuracy and sometimes are dependent to environmental situation. In additional, this location based record the position of person directly, and the position of person is determined by neighborhood. For example if a person forget and leave location based device in a train. This system record mistakes his/her position.

It may be not obvious to user that a location based device when collected information about personal position, but if user was aware of this information, could be control place and time of information collecting $[1,11]$.

\section{CONCLUSION}

We could introduce LBS to development of many science, business, jobs and etc by attention to increasingly need of human. Markets and even could be the causes of rescuing economical countries like we consider LBS discussion, such as general principle and their component and positioning. Data processing and other related topics in the presented paper.

\section{REFERENCES}

[1] Barkuus, Louise, Intel Corporation, 2003, Location Based Services for Mobile Telephony

[2] Steiniger, Stefan, Moritz Neun and Alistair Edwardes, Foundations of Location Based Services

[3] Wang, Shu, Jungwon Min and Byung K. Yi, IEEE international conference on communication (ICC) 2008, Beijing, China; Location based services for mobiles: technology and standards.

[4] Schiller, Jochen and Voisard Agnes, Morgan Kaufmann is an imprint of Elsevier 2004, location-based_services.

[5] Trevisani, Emiliano and Vitaletti Andrea, Cell-ID location technique, limits and benefits : an experimental study

[6] Quigley, Aaron, Belinda Ward, Chris Ottrey, Dan Cutting and Robert Kummerfeld,a privacy centric location aware system

[7] Aphrodite Tsalgatidou, Jari Veijalainen, Jouni Markkula, Artem KatasonovandStathes Hadjiefthymiades. , Mobile E Commerce and Location Based Services: Technology and Requirement

[8] Cebrowski, A., and J.J. Garstka, "Network Centric Warfare: Its Origins and Future", Proceedings of the Naval Institute

[9] Alberts, D.S., J.J. Ganske, F.P. Stein, Network Centric Warfare, Second Edition (Revised), CCRP

[10] PAILlARD, J. and M. BROUCHON, Active and passive movements in the Calibration of position sense.

[11] Location Technologies, tic bobile

[12] Ramamoorthy, Anand, India2004, New approaches in GPS based location systems Map

[13] Zipf, Alexander and Rainer Malaka, Developing Location Based Services for Tourism

[14] http://en.wikipedia.org/wiki/Location-based_service, accessed (10 May, 2011)

[15] Rerrer, Ulf and Odej Kao, Suitability of Positioning Techniques for Location-based Services in wireless LANs

[16] Flexible positioning for location-based service

[17] Ríos, Sergio, Location Based Services: Interfacing to a Mobile Positioning Center

[18] Montilla Bravo, Alberto, Jose Ignacio Moreno, Ignacio Soto, Advanced positioning and location based services in $4 \mathrm{G}$ mobile-ip radio access networks 